\title{
A multiobjective approach for identifying protein complexes and studying their association in multiple disorders
}

\author{
Sanghamitra Bandyopadhyay ${ }^{1}$, Sumanta Ray², Anirban Mukhopadhyay ${ }^{3}$ and Ujjwal Maulik ${ }^{4^{*}}$
}

\begin{abstract}
Background: Detecting protein complexes within protein-protein interaction (PPI) networks is a major step toward the analysis of biological processes and pathways. Identification and characterization of protein complexes in PPI network is an ongoing challenge. Several high-throughput experimental techniques provide substantial number of PPIs which are widely utilized for compiling the PPI network of a species.

Results: Here we focus on detecting human protein complexes by developing a multiobjective framework. For this large human PPI network is partitioned into modules which serves as protein complex. For building the objective functions we have utilized topological properties of PPI network and biological properties based on Gene Ontology semantic similarity. The proposed method is compared with that of some state-of-the-art algorithms in the context of different performance metrics. For the purpose of biological validation of our predicted complexes we have also employed a Gene Ontology and pathway based analysis here. Additionally, we have performed an analysis to associate resulting protein complexes with 22 key disease classes. Two bipartite networks are created to clearly visualize the association of identified protein complexes with the disorder classes.
\end{abstract}

Conclusions: Here, we present the task of identifying protein complexes as a multiobjective optimization problem. Identified protein complexes are found to be associated with several disorders classes like 'Cancer,' 'Endocrine' and 'Multiple.' This analysis uncovers some new relationships between disorders and predicted complexes that may take a potential role in the prediction of multi target drugs.

Keywords: Protein-protein interactions, Protein complex, Multiobjective optimization, Gene Ontology, Disorders

\section{Background}

Recent advancement in biotechnology produces lots of information about protein-protein interactions. Those information act as a potential source to construct the protein-protein interaction network (PPIN) for a single species. Protein complexes are generally described as molecular aggregation of a set of proteins connected by multiple protein-protein interactions. Protein complexes play different functions in the cell. It can serve as cellular machines, rigid structures, and posttranslational modification systems. In general cellular functions and

\footnotetext{
*Correspondence: umaulik@cse.jdvu.ac.in

${ }^{4}$ Department of Computer Science and Engineering, Jadavpur University, Kolkata 700032, West Bengal, India

Full list of author information is available at the end of the article
}

biochemical events in cell are coordinately performed by a groups of proteins interacting with each other in protein complexes. Identifying such protein complexes is important for understanding the structure and functions of these biochemical events. Moreover, changing of interaction pattern of proteins is the consequence of many diseases. Identifying such interactions through protein complexes predominantly lead to applications in disease diagnosis.

In usual representation, protein complexes take the form of a dense clusters of proteins connected through multiple interactions. Different computational methods for finding dense regions in the PPI network are available in literature. Several techniques based on graph clustering, finding dense regions, or clique finding have been 
proposed to discover protein complexes in PPI networks [1-4]. In [5] Molecular Complex Detection (MCODE) has been proposed to detect protein complexes in PPI network. MCODE generally emphasizes on the local neighborhood density of nodes and puts weight to all the nodes corresponding to the local density. Starting from the top weighted node it iteratively adds vertices which have weights above a certain threshold.

In [6] a clustering with overlapping neighborhood expansion (ClusterONE) is proposed for detecting overlapping protein complexes from protein-protein interaction data. ClusterONE generally follows a greedy procedure to update the partially constructed groups of vertices based on high cohesiveness among the vertices. The growth process is repeated from different seeds to form multiple, possibly overlapping groups. In the second step, overlapping groups are merged based on overlap scores.

In [7] an algorithm called Affinity Propagation is proposed which is basically an unsupervised algorithm and thus the number of clusters is automatically calculated. The data points are grouped based on the similarity between each pair of data points. Initially all data points are considered as potential "exemplars". The main algorithm is concentrated on finding sub-paths, which allow easy message exchanges between nodes. In subsequent steps, the exchange of message is continued to happen between the nodes until a set of exemplars and corresponding clusters with high quality come out.

In [8] a multiobjective framework is proposed for detecting protein complexes in yeast PPI network. Here two different types of objectives are utilized for searching over the whole PPI network to predict modules which serve as protein complexes. Density of the module and Gene Ontology based semantic similarity measures are taken into account for building the objectives.

All these methods primarily focus on the detection of protein complexes in PPI network of model organism yeast (S. cerevisiae). Although, there have been several studies on determining and analyzing protein complexes in a single organism, computational analysis of human protein complexes is not studied in extensive manner. Some studies have analyzed human protein complexes based on a particular disease association [9-11]. These studies are focused on finding protein complexes associated with some specific disease. Here, we have proposed a multiobjective evolutionary technique for detecting protein complexes in human PPI network and studied their involvement in different disease classes.

In general, it has been observed that the proteins within a complex are functionally similar and carry out common biological activities. For measuring the functional similarity between two proteins we have computed semantic similarity between Gene Ontology terms associated with those proteins. In linguistic, to measure the similarity between two concepts, semantic similarity is used. This can be extended to measure the similarity between GO-terms in the GO database [12]. Here, we have utilized the Relevance [13] semantic similarity measure for obtaining semantic similarity between two proteins. This serves one of the objective function of our multiobjective framework. Besides the semantic similarity measure topological properties of PPI network are also used here for building the objective functions. Non dominated sorting genetic algorithm II (NSGA-II) [14, 15], a popular multiobjective Genetic Algorithm (GA) [16] has been utilized as the underlying optimization tool. The results are collected by applying the proposed algorithm in the protein-protein interaction (PPI) data downloaded from the Human Protein Reference Database (HPRD) [17]. The performance of our method is compared with that of some other existing methods such as MCODE [5], clusterONE [6], Affinity propagation [7], Core attachment method [18], COACH [19], RNSC [20], MCL-Caw [21], and PEWCC [22].

Here, we have reported the associations among predicted complexes with similar type of diseases/disorders. Identifying the associations between human protein complexes and multiple disorders is essential for understanding disease mechanism and is also important to assist drug developers for the development of new diagnostics and therapeutics. In Goh et al. [23], a bipartite network is formed that shows disorder-gene association which lead to the concept of 'diseaseome'. In this network one set of nodes represent all known genetic disorders, and the other set corresponds to all known disease genes in the human genome. A disorder and a gene is connected by a link if the mutations in that gene is incriminated by that disorder. They found that genes associated with similar diseases have an increased tendency to interact with one another, and tend to exhibit high connectivity with each other forming a dense cluster. So it is necessary to discover the association of our predicted protein complexes with those genes causing similar diseases. For this purpose we have analyzed the predicted complexes and associated them with genes causing similar disease. We have searched the involvement of proteins within predicted complexes in 22 type of primary disease/disorder classes and found most of them are associated with 'Cancer' disease class. We have also formed two bipartite networks between all predicted protein complexes and disease/disorder classes. These networks show the involvement of protein complexes within disorders/ disease classes. This may uncover interesting association or relationship between diseases and protein complexes. This can contribute significant effort to develop new 
strategies in Polypharmacological drug discovery which focus on multi-target drugs.

\section{Methods}

In this section we describe the proposed multiobjective method for detecting protein complexes in human PPI network. Non-dominated Sorting GA (NSGA-II) [14] is employed as an underlying multiobjective framework.

\section{Chromosome representation}

Here a protein complex (or a subgraph of human PPI graph) is encoded as a chromosome. It is represented as $p_{1}, p_{2} \ldots p_{n}$ where $p_{i}$ is the ith protein in whole human protein set. Thus a chromosome represents a protein complex containing the nodes $p_{1}, p_{2}, \ldots p_{n}$, and the edges among them represent interactions.

\section{Population initialization}

For starting from a reasonable position we construct the initial population as a set of modules with high density. For this purpose we randomly chose some substructure consisting of all 1s from adjacency matrix. To find out all 1s substructures we apply a biclustering technique [24] and randomly pick up some of the biclusters consisting of all 1s. Here, union of rows and columns of each bicluster is treated as a chromosome, which comprise the initial population.

\section{Representation of objective functions}

Here two categories of objective functions are built, one is based on the topological characteristics of the network and the other captures gene ontological similarity of proteins. To define the two objective functions belonging to the first one we have incorporated some graphbased properties of the PPIN. For the other category, we employed GO based semantic similarity measure.

\section{Objective functions related to the topological properties}

We have defined two objective functions in this category. One is based on the density of protein complexes, and other is based on closeness centralities. The density of a graph is defined as ratio of the number of edges present in a graph to the possible number of edges in a complete graph of same size. Protein complexes generally represent high dense area in the PPIN. Large number of interactions (or edges) among proteins (or nodes) in the complex is the possible reason behind that. Thus, using density as an objective function and maximizing it for individual subgraphs will yield much denser complexes.

For choosing the next objective we calculate the contribution of a node as follows: $\operatorname{Contr}\left(n_{i}\right)=\frac{\left|N_{n_{i}}\right|}{\operatorname{degree}\left(n_{i}\right)}$, where $N_{n_{i}}$ represents the set of nodes directly connected with node $n_{i}$ in a protein cluster $C$. Now the contribution of a protein cluster can be calculated as the summation of these values which can be formulated as:

$$
\sum_{n_{i} \in C} \operatorname{Contr}\left(n_{i}\right)
$$

Maximizing this will produce clusters having small number of outward interaction partners for a node, thus producing compact as well as separated clusters.

Closeness centrality of a vertex in a graph is defined as the reciprocal of average shortest-path distance to other vertices. It can be considered as the efficiency of a node (individual) in spreading information to others in the network. Higher value of it indicates that most of the nodes are closer to that node. Here we maximize the objective function:

$$
\sum_{n_{i} \in C} C C\left(n_{i}\right)
$$

where $C$ represents a protein cluster and $C C\left(n_{i}\right)$ is the closeness centrality of the node $n_{i}$. Maximizing this ensures that the resulting clusters have nodes which are more central in the whole protein interaction graph and are likely to form a protein complex.

\section{Objective function related to Gene Ontology}

Proteins within the protein complexes are functionally similar to each other. This suggests that these proteins have high semantic similarity among themselves. This is measured by computing the semantic similarity between GO-terms they are annotated with. As proteins are annotated with multiple GO terms, so, the similarities are calculated by averaging the similarities of the GO term cross pairs which are associated with them [25]. We have calculated the similarities among all pairs of proteins in the PPI network and given these as weights of edges in semantic similarity network. The average similarity of all pairs of proteins corresponding to the edges of a chromosome is treated as fitness value of it. For example, the fitness of a chromosome or a subgraph is calculated by summing up all the weights of edges and averaging these values. This can be written as:

$$
\operatorname{sim}(s)=\frac{\sum_{i=1, j=1, i \neq j}^{p} w\left(n_{i}, n_{j}\right)}{p},
$$

where $s$ is the chromosome consisting of nodes $n_{1}, n_{2}, \ldots n_{p},\left(n_{i}, n_{j}\right) \epsilon E$, where $E$ is the set of edges and $w$ is a weight function defined as $w: E \rightarrow[0,1]$.

\section{Mutation procedure}

The usual genetic operators are selection, crossover, and mutation. Here crossover operation is not performed as it produces large number of disconnected graphs. For 
selection the conventional crowded binary tournament selection in NSGA-II is used here. As whole subgraph is encoded as a chromosome, so a perturbation in node is performed by means of mutation with a high probability $(p=0.9)$.

If a chromosome $n_{1}, n_{2}, \ldots, n_{9}$ is selected to be mutated then the following process is performed:

- Randomly select some of the nodes.

- Insertion and deletion are performed with equal probability:

- Insertion: Add the nodes which are directly connected with the selected nodes.

- Deletion: Delete the selected nodes.

The whole process is shown in Fig. 1.

\section{Results and discussions}

Here we illustrate the performance of our proposed technique and compare this with three well known algorithms MCODE [5], clusterONE [6] and Affinity propagation [7]. We download human PPI dataset from Human
Protein Reference Database (HPRD) [17] which contains 39,240 interactions among 9,589 human proteins (The data is given in Additional file 1). Table 1 summarizes the topological properties of the network created from this database. We compare the results with known protein complexes downloaded from a database PCDq [26]. It consists of both predicted and curated human protein complexes and is contains 1,264 complexes with 9,268 proteins and 32,198 PPIs. To investigate the functional enrichment of our predicted complexes we have also performed a Gene Ontology based analysis here. The source code of our proposed method is given in Additional file 2.

\section{Performance comparisons with existing methods}

Here, for comparing our results with that of some stateof-the-art algorithms we have utilized some matching statistics like Sensitivity, Positive Predictive Value (PPV), and Accuracy [27].

We have built a Contingency Table $(T)$ with $n$ rows and $m$ columns where rows and columns represent predicted and real protein complexes, respectively. The value of each cell $T_{i, j}$ indicates the number of common proteins between real and predicted complexes.

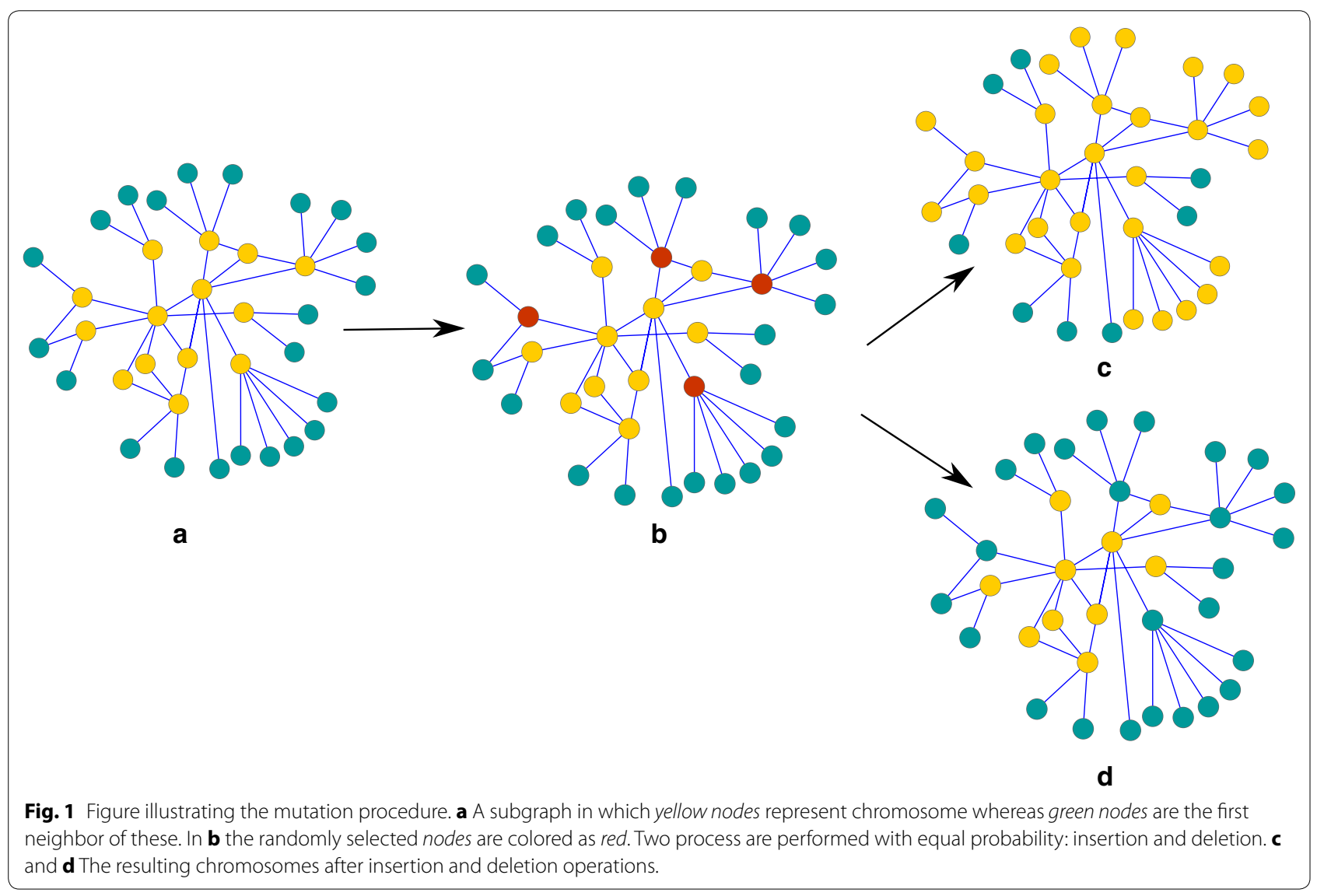


Table 1 Summary of the human PPI network data sets used here

\begin{tabular}{llllllllll}
\hline Data set & \#Proteins & \#Interactions & Avg. degree & Max. degree & Density & $\begin{array}{l}\text { Clustering coef- \#Connected } \\
\text { ficient }\end{array}$ & $\begin{array}{l}\text { Network diam- Avg. number } \\
\text { components }\end{array}$ & ef neighbors \\
\hline HPRD & 9,589 & 39,240 & 7.924 & 271 & 0.001 & 0.102 & 262 & 14 & 7.703 \\
\hline
\end{tabular}

\section{Sensitivity}

Sensitivity is defined as the fraction of proteins in real complex $i$ found in predicted complex $j: S n_{i, j}=\frac{T_{i, j}}{N_{i}}$, where $N_{i}$ is the number of proteins belonging to complex $i$. A complex-wise sensitivity $S n_{c o_{i}}$ is defined as: $S n_{c o_{i}}=\max _{j=1}^{m} S n_{i, j}$. The General Sensitivity $\left(S_{n}\right)$ is the weighted average of complex-wise sensitivity $S n_{c o_{i}}$ over all complexes and is defined as:

$$
S_{n}=\frac{\sum_{i=1}^{n} N_{i} S n_{c o_{i}}}{\sum_{i=1}^{n} N_{i}} .
$$

\section{Positive predictive value}

The positive predictive value is the proportion of proteins in predicted complex $j$ which belong to the real complex $i$ and is defined as: $P P V_{i, j}=\frac{T_{i, j}}{\sum_{i=1}^{n} T_{i, j}}=\frac{T_{i, j}}{T_{. j}}$, where $T_{. j}$ is the marginal sum of a column $j$. Complex-wise-wise positive predictive value $P P V_{c l_{j}}$ represents the maximal fraction of proteins of predicted complex $j$ found in some real complex: $P P V_{c l_{j}}=\max _{i=1}^{n} P P V_{i, j}$. The General PPV(PPV) of a clustering result is the weighted average of complex-wise $\operatorname{PPV}\left(P P V_{c l_{j}}\right)$ over all predicted complexes, and is defined as:

$$
P P V=\frac{\sum_{j=1}^{m} T_{. j} P P V_{c l_{j}}}{\sum_{j=1}^{m} T_{. j}} .
$$

\section{Accuracy}

The Geometric Accuracy (Acc) represents a tradeoff between sensitivity and the positive predictive value and it is defined as:

$$
A c c=\sqrt{S_{n} * P P V} .
$$

The advantage of taking the geometric mean is that it yields a low score when either the $S_{n}$ or the $P P V$ metric is low. High accuracy value thus requires a high performance for both the criteria.

In Table 2 we show the comparative performance of different existing algorithms with our proposed method using these three metrics. It may be noted that the proposed method performs comparatively well than the other algorithms with respect to sensitivity, PPV and accuracy.

We have also performed an analysis to compare the performance of different existing algorithm with the proposed one. Let $B=B_{1}, B_{2}, \ldots B_{n}$ and $C=C_{1}, C_{2}, \ldots C_{m}$
Table 2 Comparison of performance of different algorithms with respect to sensitivity, PPV and accuracy

\begin{tabular}{llll}
\hline Method & Sensitivity & PPV & Accuracy \\
\hline MCODE & 0.2134 & 0.5274 & 0.3347 \\
ClusterONE & 0.1414 & 0.4562 & 0.2540 \\
Affinity_propagation & 0.1837 & 0.4443 & 0.2857 \\
CORE & 0.315 & 0.353 & 0.333 \\
RNSC & 0.379 & 0.469 & 0.4216 \\
MCL-Caw & 0.331 & 0.241 & 0.279 \\
PEWCC & 0.435 & 0.469 & 0.451 \\
Proposed_Method & 0.3061 & 0.6928 & 0.4605 \\
\hline
\end{tabular}

be the sets of benchmark and predicted complexes respectively. The Jaccard index $J$ represents the overlap between a benchmark complex and predicted complex. It is defined as $J\left(B_{i}, C_{j}\right)=\frac{\left|B_{i} \bigcap C_{j}\right|}{\left|B_{i} \bigcup C_{j}\right|}$. A benchmark complex is said to be covered by a predicted complex if the value of $j$ is greater than some threshold value. In this respect Recall (coverage) and precision (sensitivity) can be defined as

$$
\text { Recall }=\frac{\left|B_{i}\right|}{|B|},
$$

where $J\left(B_{i}, C_{j}\right)>t$, for some $C_{j} \epsilon C$, and

$$
\text { precision }=\frac{\left|C_{j}\right|}{|C|},
$$

where $J\left(B_{i}, C_{j}\right)>t$ for some $B_{i} \in B$

We evaluate the performance of the existing methods by plotting the precision versus recall curves for the predicted complexes. These curves are plotted by tuning the threshold value $t$ from 0 to 1 . This is shown in Fig. 2. From this plot we have also computed the AUC score for each of the methods. The AUC score is shown in Table 3. It is evident from the Fig. 2 and Table 3 that proposed method shows best precision and recall compare to the other state-of-the-art. The processed data for constructing the ROC plot are given in Additional file 3.

\section{Analysis of predicted complexes}

In Table 4 we have shown the resulting protein complexes and compared them against the real one. Most of 


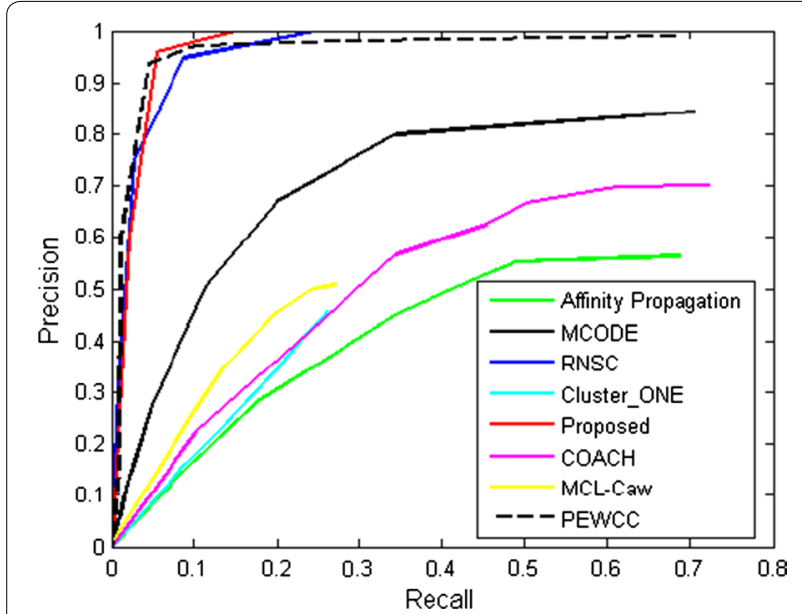

Fig. 2 Precision vs recall curves for all methods at different threshold values ( $\mathrm{t}$ ).

them show a good overlap with real complexes. We plot a bar diagram to show this overlap. In Fig. 3 Y-axis represents proportion of proteins in predicted complexes involved in some real one. From this figure it is noticeable that most of the protein complexes share good proportion of proteins with some real complexes with an average proportion of $79.68 \%$. The smallest complex consists of 11 proteins in which 8 proteins are involved in some real complexes whereas the largest one comprises of 272 proteins out of which 183 proteins are involved in some real complexes. We have also preformed a GO and pathway based study to biologically validate the predicted complexes.

Columns 3 and 4 of Table 4 represent predicted complexes and the list of proteins that are matched with some real one, respectively. Columns 5, 6, 7 and 8 of this table represent most significant GO-terms with three annotations [viz., biological process (BP), cellular component (CC), molecular function (MF)] and KEGG pathways that are associated with the predicted complexes. Here, we notice that more than one complex are grouped in one predicted cluster. For example in row 4 of Table 4 the predicted cluster captured two complexes: $\mathrm{p} 300-\mathrm{MDM} 2-\mathrm{p} 53$ protein complex and CDH1CTNNB1-PTPN1 complex. It is not quite unexpected because the real complexes that have some common functionality or have some common signaling pathway tend to group in one cluster. In row 1, predicted cluster 5 captures good proportion proteins of four real complexes viz., CTNNB1-DVL1-DVL3-PPM1A complex (80\%), HSPB1-PPA1-PPA1-SETDB1-TP53-WIPI1 complex (66.67\%), EEF1A1-MDH2-WARS complex $(66.67 \%)$ and transforming growth factor-SMAD complex (66.67\%). The complex CTNNB1-DVL1-DVL3PPM1A is composed of genes DVL1, DVL2, CTNNB1, PPM1A and DVL3. Protein phosphatase 1A (PPM1A) is an enzyme which is encoded by the PPM1A gene. The proteins encoded by this gene is a member of PP2C family of Ser/Thr protein phosphatases and are generally known to be a negative regulator of cell stress response pathways. Catenin (cadherin-associated protein), beta 1 (CTNNB1) is an integral part of the canonical Wnt signaling pathway which is a network of proteins that passes signals from receptor of the surface of cell to the nucleus that leads to the expression of target genes. Signaling via Wnt signaling pathway also causes activation of histone methyltransferase (SETDB1) and subsequently represses PPARgamma transactivation [28]. Moreover SMAD1 indirectly enhances Wnt signaling by suppressing the expression of Wnt signaling inhibitors [Dickkopf 1 (Dkk1) and 2] with interleukin (IL)-11. We can notice from Table 4, row-1 that predicted cluster 5 is associated with Colorectal cancer pathway (p-value: $2.9 \mathrm{E} 11$ ). In different literature [29-31] it is established that activation of the Wnt signaling pathway via mutation of the adenomatous polyposis coli gene (APC) is the critical reason for colon carcinogenesis.

Most of our predicted protein complexes are associated with SMAD complexes like transforming growth factor-SMAD complex, FOXO-SMAD complex, SMAD1SMAD4-ECSIT2 containing complex etc. SMADs are intracellular proteins that transduce extracellular signals from transforming growth factor beta (TGF- $\beta$ ) ligands to the nucleus. In the nucleus, SMAD complexes attach in some specific areas of DNA and control the activity of particular genes and regulate cell proliferation [32].

From Table 4 it is worth-mentioning that most of the predicted clusters are enriched with several cancer related pathways viz., colorectal cancer, chronic myeloid leukemia, prostate cancer etc. This suggests that the predicted clusters are biologically meaningful and important for uncovering different cancer associated modules.

Table 3 AUC score of different methods

\begin{tabular}{lllllllll}
\hline & MCODE & ClusterONE & Affinity_Propagation & COACH & RNSC & MCL-Caw & PEWCC & Proposed method \\
\hline AUC & 0.1096 & 0.0590 & 0.2714 & 0.3572 & 0.2277 & 0.0841 & 0.6595 & 0.7244 \\
\hline
\end{tabular}




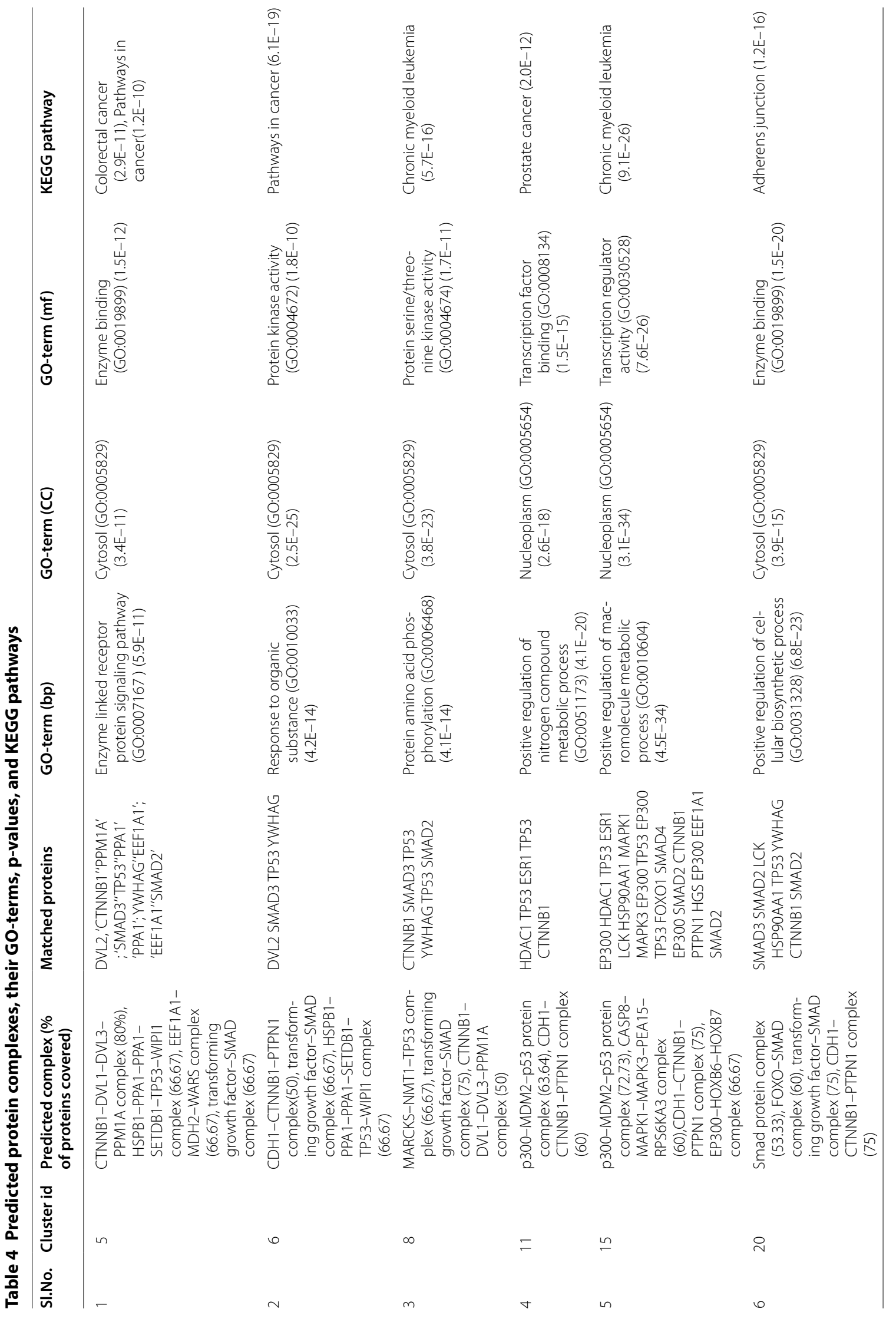




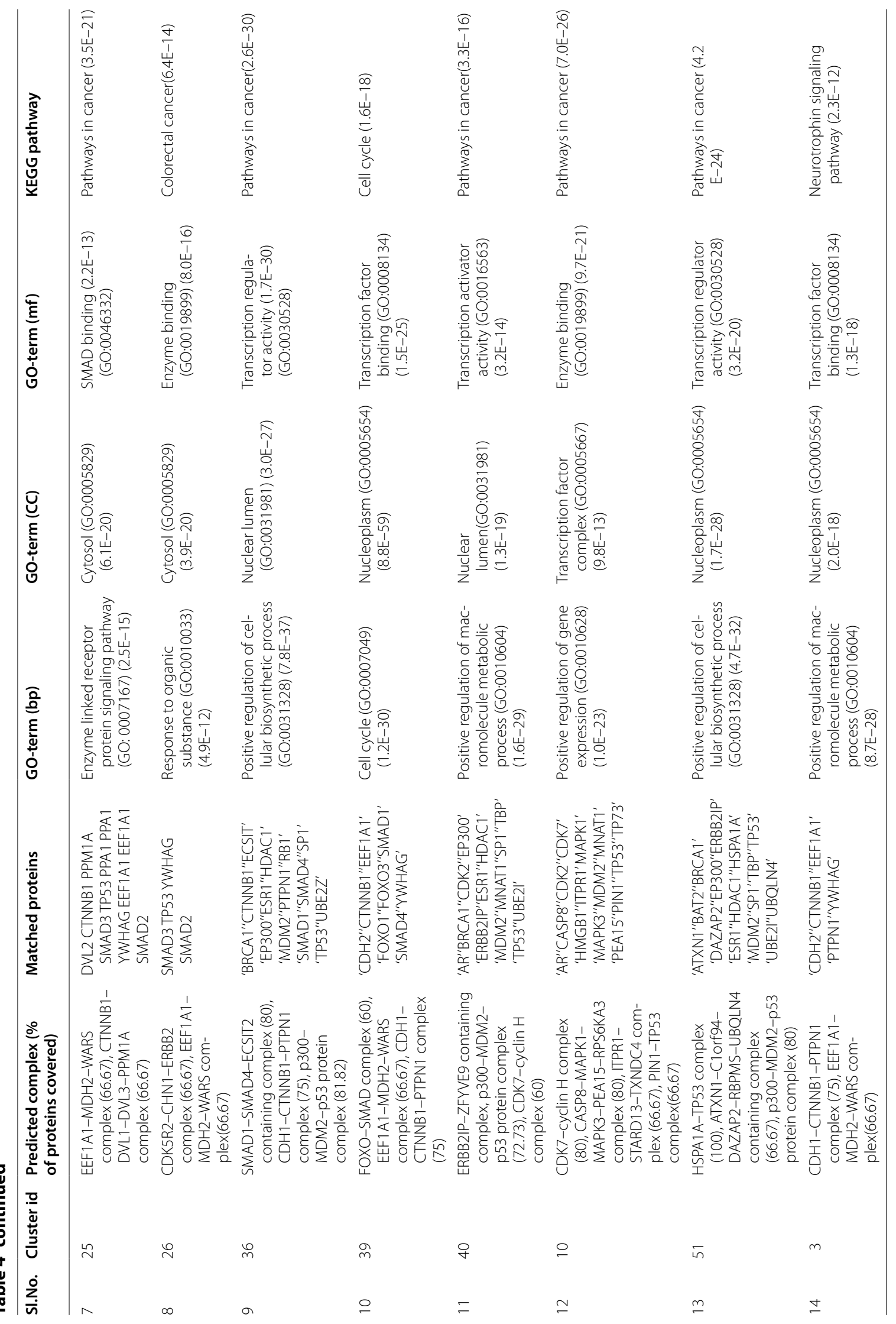




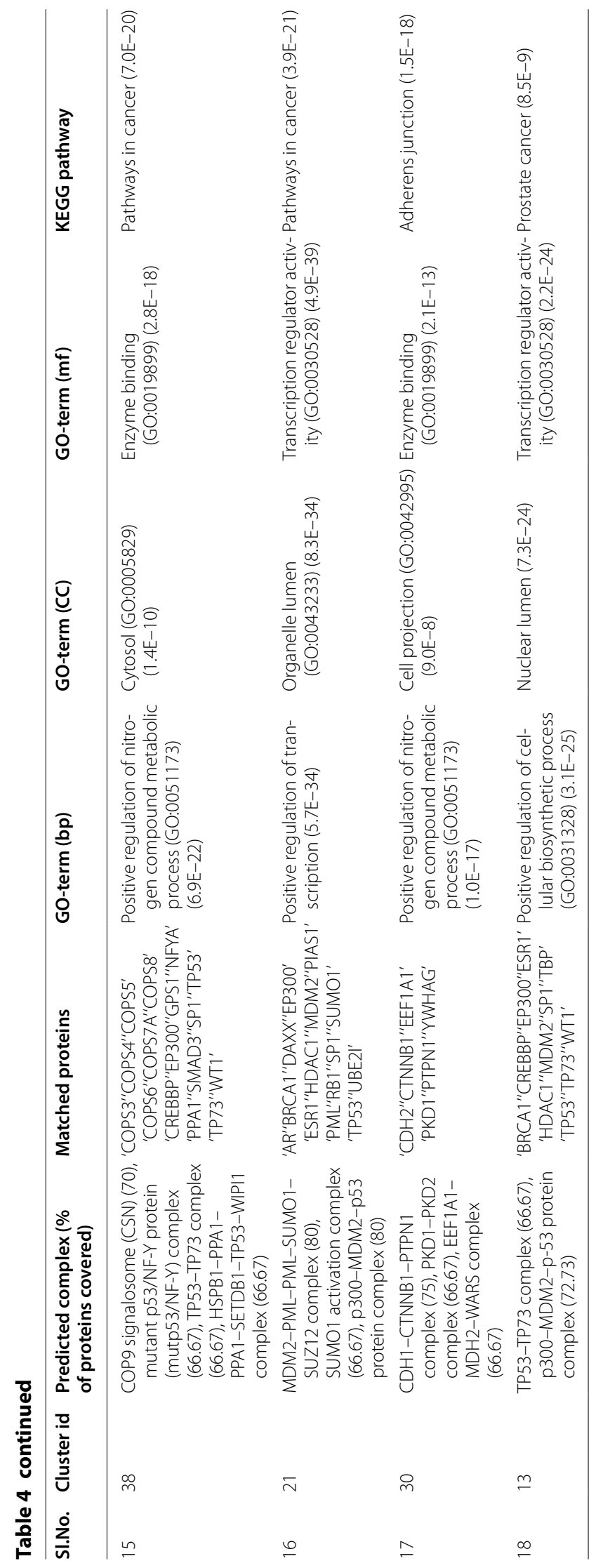




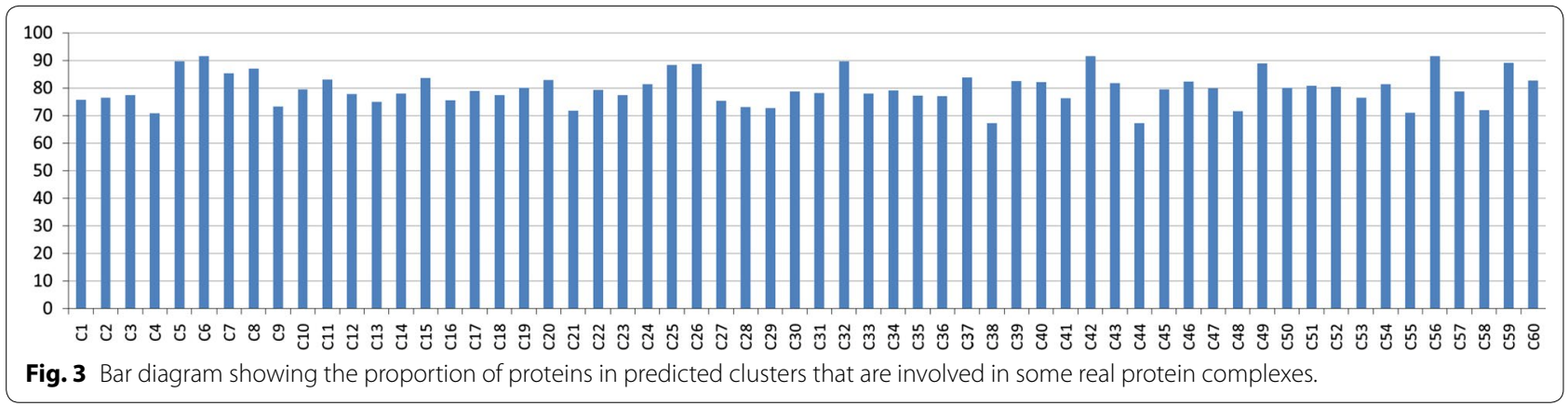

Association of predicted complexes in disorders/diseases Here, we discuss the involvement of predicted protein complexes in different disease/disorder classes. The list of disorders/diseases, disorder associated genes and association between genes and disorder/disease classes are obtained from Goh et al. [23]. In Goh et al., a classification of disorders can be found depending on the physiological system affected by the disorder. They have classified all genetic disorders in 22 primary classes and associated all the genes corresponding to all the disorders. In each class there is a list of disorders/diseases that exhibit similar type of clinical features affected by these disorders. Here, we find an association of our predicted complexes with these 22 disorder/disease classes. To test the biological plausibility of the identified complexes we draw a bipartite network between protein complexes and 22 disorders/diseases to find disease associated complexes. We have also tested the involvement of proteins belonging to our predicted complexes in those disorders.

\section{Involvement of identified complexes in 22 primary disorders/ disease classes}

To show to what extent the proteins of our predicted complexes are involved in specific disorder/disease classes we plot a bar diagram showing the proportion of protein complexes involved in each of the disorder classes. We assume that a protein complex is associated with specific type of disorder if all the proteins associated with this disorder are belonging to that protein complex. The bar diagram is shown in Fig. 4. From this figure we can notice that subsequent number of protein complexes are associated with 'cancer' and 'multiple' disease classes. 'Cancer' class consists of 113 disorders whereas 'multiple' class contains 155 disorders. The disorders are assigned in each class based on the similarity of clinical properties of these disorders and the observation of physiological system mostly affected by those disorders. Disorders having multiple clinical features are placed in the 'multiple' class.

We observe from Fig. 4 that most of the predicted complexes are enriched with proteins that are implicated by different disorders involved with 'cancer' and 'multiple' disorder classes. Some proteins of our predicted complexes are also involved in the disorders associated with 'connective tissue', 'Developmental' and 'Endocrine' classes. We have been able to associate the predicted complexes with 15 classes of disorders amongst 22 disorder classes.

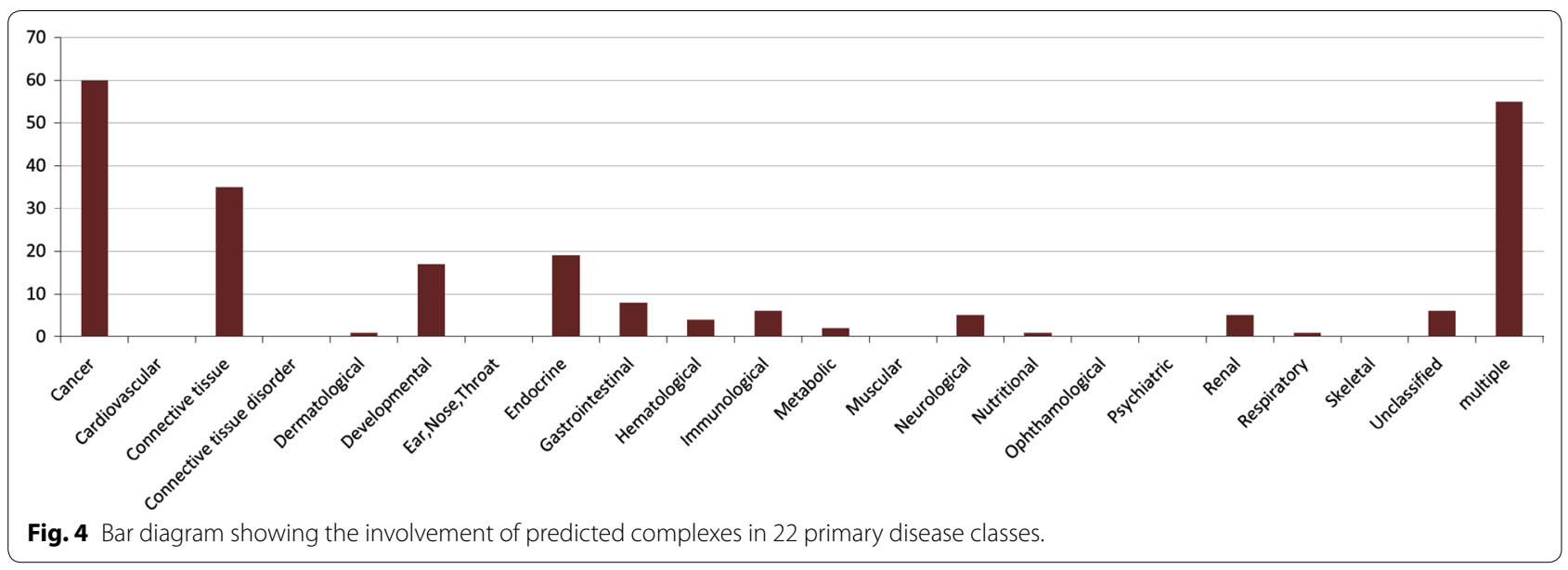




\section{Complex-disease bipartite network}

To identify the overall association between predicted complexes and disease classes we have created a bipartite network between predicted protein complexes and the associated disease classes. One partite set constitutes the complexes, whereas other partite set represents the associated disease classes. Each partite set is connected with other by edges depending on the association between predicted complexes and the disorders involved in disease classes. The network is shown in Fig. 5. The predicted complexes are represented by red nodes whereas the disease classes are denoted by yellow diamond shaped nodes. It is possible that several proteins in one complex are involved in several disorders belonging to different disease classes. So, we have calculated the number of disorders associated with each predicted complex. We say a disorder is associated with a predicted complex if all the proteins associated with that disorder is involved in that complex. To show to what extent the protein complexes are associated with disorders we vary the size of red nodes based on the total number of disorders associated with those complexes. An edge between a complex and a disease class indicates the association of disorders belonging to the disease class with that complex. Edge width indicates the number of associated disorders with the corresponding complex and disease class

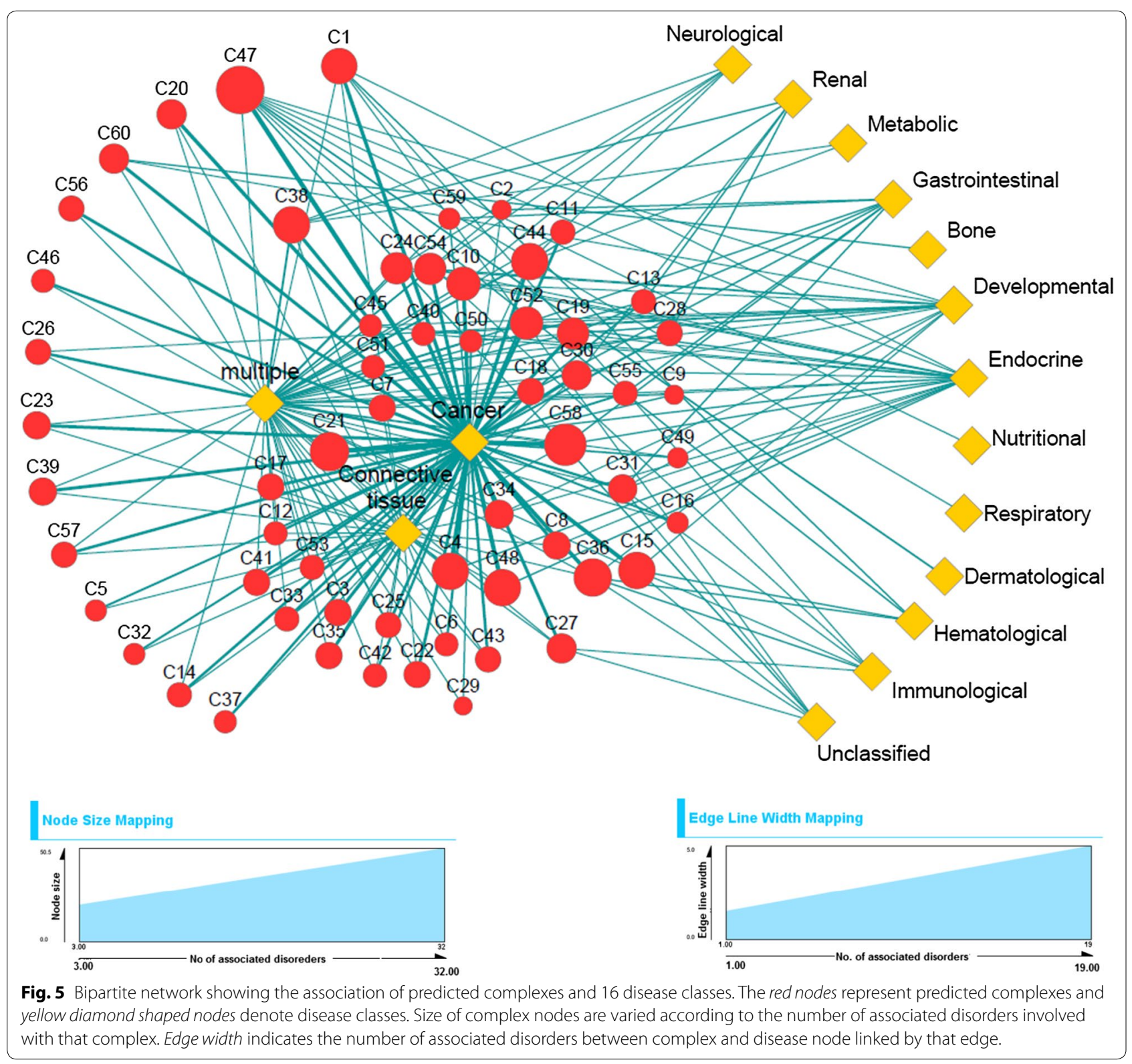


linked by that edge. It appears from Fig. 5 that most of the complexes are associated with significant number of disorders belonging to different disease classes. Interestingly it is found that all the complexes are more or less associated with 'cancer' related disorders. It suggests that the predicted complexes are enriched with proteins that are involved in different cancer related disorders and may be considered as important candidates to uncover different associations for understanding disease mechanisms, diagnosis and therapy. Other disorders like 'Connective tissue', 'multiple' and 'Endocrine' also show reasonable amount of association with different complexes. In Additional file 3 the processed data for constructing the complex-disease bipartite network are given.

We have also performed an analysis to describe the association among protein complexes and disease classes. For this purpose, we have collected PPI information and GO based semantic similarity information of all the genes associated with 22 disease classes. The PPI and GO semantic similarity informations are subsequently converted into $22 \mathrm{PPI}$ and GO semantic similarity networks.

We have computed density and average semantic similarity scores from these networks and showed in Table 5. First and second column of the table represent disease category and number of associated genes in it, respectively. The third column shows number interactions among the genes, in each category. The last two columns represent density and average semantic similarity score of interaction network. It is noticeable that among all disease classes cancer associated proteins have better score than others. The predicted complexes are functionally similar, and it may be a possible reason for the overrepresentation of cancer associated genes in predicted complexes. For the similar reason, other disorders like 'Connective tissue,' 'multiple' and 'Endocrine' have better amount of association with different complexes.

To identify the association among disorders and protein complexes, we have also created another bi-partite network. As depicted in Fig. 6, the network is composed of two types of nodes. The big red nodes represent predicted complexes and small nodes stand for different disorders. Out of 22 disease classes we have found 16 classes have different disorders associated with the predicted complexes. In Additional file 4: Table S1 we have listed all the disorder names associated with specific complexes. 1st column of the Table S1 (Additional file 4) represents predicted protein complex, whereas the second and third columns represent the associated disorders and the corresponding disease class. From this table we can notice that the proteins in complex 1 is associated with 11 'cancer' associated disorders, 3 hematological disorders, 1 'Endocrine,' 'Connective tissue,' and 'Immunology'

Table 5 Some useful metrics of disorder associated genes

\begin{tabular}{|c|c|c|c|c|}
\hline Disease category & No. of associated genes & No. of interaction in human PPI & Density & Semantic similarity \\
\hline Bone & 180 & 94 & 0.0163 & 0.5676 \\
\hline Cancer & 869 & 725 & 0.0019 & 0.8931 \\
\hline Cardiovascular & 332 & 457 & 0.0083 & 0.7374 \\
\hline Connective_tissue & 149 & 75 & 0.0068 & 0.7146 \\
\hline Dermatological & 270 & 154 & 0.0042 & 0.6381 \\
\hline Developmental & 143 & 219 & 0.0216 & 0.7589 \\
\hline Ear, nose, throat & 177 & 183 & 0.0117 & 0.8277 \\
\hline Endocrine & 305 & 572 & 0.0123 & 0.8432 \\
\hline Gastrointestinal & 110 & 52 & 0.0087 & 0.5819 \\
\hline Hematological & 389 & 755 & 0.0100 & 0.6449 \\
\hline Immunological & 290 & 658 & 0.0157 & 0.8159 \\
\hline Metabolic & 638 & 2,088 & 0.0103 & 0.7458 \\
\hline Muscular & 294 & 1,183 & 0.0275 & 0.6421 \\
\hline Neurological & 917 & 898 & 0.0021 & 0.7811 \\
\hline Nutritional & 51 & 82 & 0.0643 & 0.6536 \\
\hline Ophthalmological & 563 & 555 & 0.0037 & 0.7035 \\
\hline Psychiatric & 75 & 49 & 0.0177 & 0.7032 \\
\hline Renal & 170 & 131 & 0.0091 & 0.6545 \\
\hline Respiratory & 79 & 1 & 0.00032 & 0.7023 \\
\hline Skeletal & 278 & 186 & 0.0048 & 0.6679 \\
\hline Unclassified & 64 & 6 & 0.0030 & 0.5080 \\
\hline Multiple & 721 & 427 & 0.0016 & 0.8019 \\
\hline
\end{tabular}




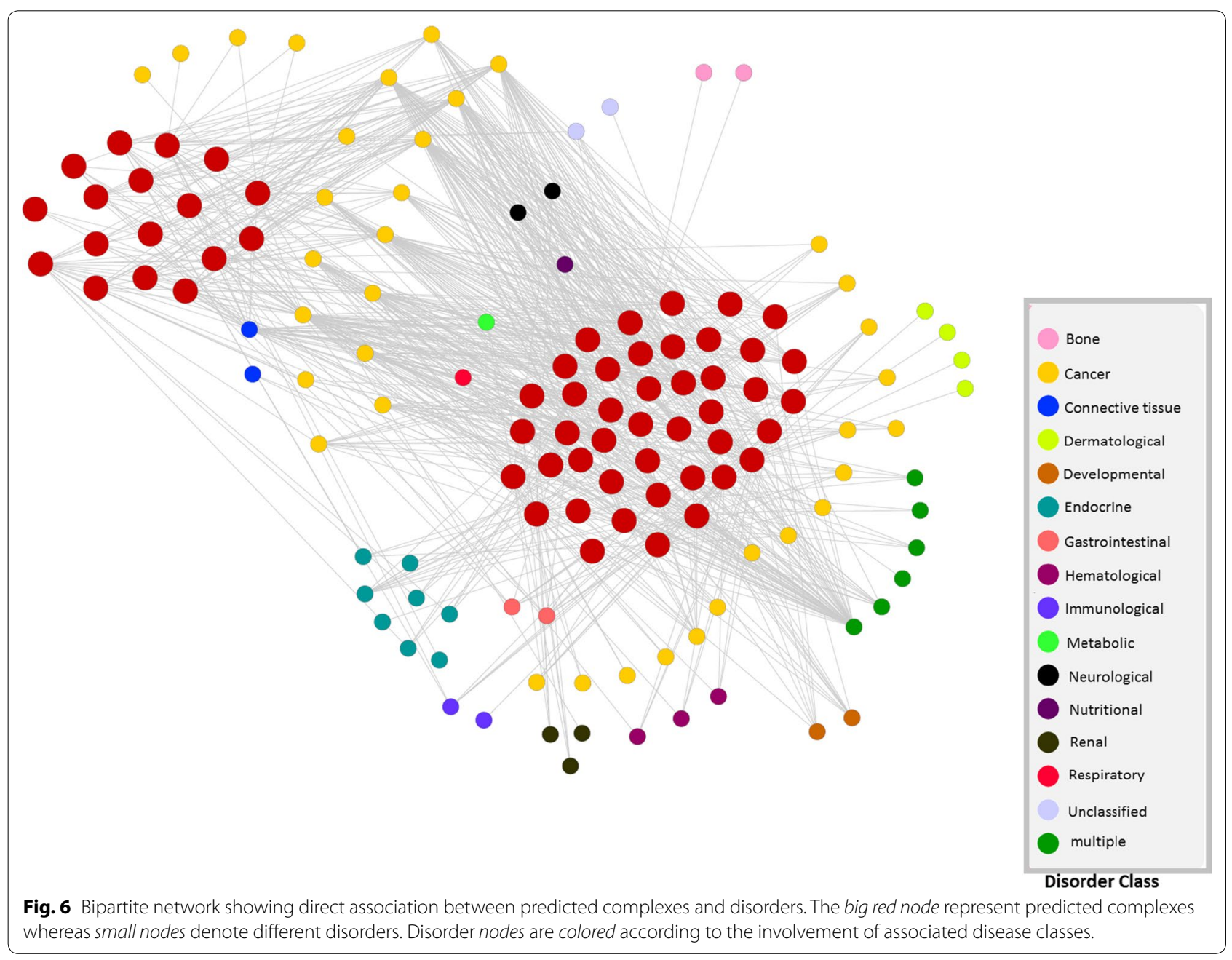

related disorders. All the proteins implicated by those disorders are clustered in complex 1 . This suggests that these associated disorders of different disease classes are loosely related with each other. For example 'Leukemia, acute promyelocytic disorder' may be developed by a long course of 'Polycythemia vera' which is associated with 'Hematological' disorders [33, 34]. From the Table S1 (Additional file 4) we can notice that the disorder 'Pilomatricoma' which belongs to the 'Cancer' disease class and the disorder 'Rubinstein-Taybi syndrome' related to the 'multiple' disease class are involved in complex 1. In [35] an abnormal association between multiple perforating and non-perforating pilomatricomas with Rubinstein-Taybi syndrome are reported. From Table S1 (Additional file 4) it can also be observed that the disorder 'Thrombocythemia, essential' involved in 'Hematology' disease class is grouped with disorder 'Renal cell carcinoma' belonging to 'Cancer' disease class in complex 1. This suggests that these two disorders are somehow related based on topological and ontological properties of the proteins that are directly associated with those disorders. It is because all the proteins implicated by these two disorders are grouped in the same complex. Interestingly in [36] a statistical correlation is also observed between essential Thrombocythemia and the survival of surgically treated renal cell carcinoma patients.

From Table S1 (Additional file 4) we can see that complex 4 is associated with 12 cancer related disorders, 3 endocrine disorders and 1 multiple disorder. The predicted complex $\mathrm{C} 4$ contains all the proteins which are related with 'Hyperparathyroidism' disorder and 'Colorectal Cancer' disorder. 'Hyperparathyroidism' is a disorder in which parathyroid (PTH) glands are overactivated and produces excess $\mathrm{PTH}$ hormone in our body. It is reported that primary hyperparathyroidism (PHP) is associated with malignancy and decreased intracolonic calcium $(\mathrm{Ca})$ that plays a role in colorectal carcinogenesis [37]. We can notice that disorder 'Parathyroid adenoma' is also associated with complex C4. A 'parathyroid adenoma' is a noncancerous (benign) tumor of the parathyroid glands but 
in many cases it is reported that it causes 'Hyperparathyroidism' [38, 39]. 'Rabson-Mendenhall syndrome' in 'multiple' disease class is a rare genetic disorder mainly caused by mutation of insulin receptor gene 'INSR'. It appears from the Table S1 (Additional file 4) that this disorder is associated with about eight predicted complexes. Most of these associated complexes also contain other disorders which are belonging to different disease classes and are linked with extreme insulin resistance due to mutations in the insulin receptor gene 'INSR'. For example complex C7 is associated with disorder 'Leprechaunism' [commonly known as Donohue syndrome (OMIM 246200)] which is a latent inherited disorder and is caused by defect of insulin receptor genes. This suggests that although the disorders 'Rabson-Mendenhall syndrome' and 'Leprechaunism' are belonging to different disease classes but the genes responsible for these disorders exhibit substantial amount of similar functional information. The possible reason behind this is that these two disorders show strong inclination in being grouped in most of the complexes.

In most of the cases we observe that similar type of disorders have a tendency to get involve in same protein complexes. So we can conclude that protein complexes not only provide a better understanding in molecular evolution but it can also unveil several information of human disorders and uncover new strategies for therapeutic intervention. This may lead to development of new potential strategies to deal with key diseases by giving more importance in protein complex formation information rather than targeting individual proteins.

\section{Conclusions}

This study introduces a multiobjective approach for detection of protein complexes in human PPI network. Integrating topological features along with $\mathrm{GO}$ features, we are able to group functionally similar proteins in same clusters which serve as protein complexes. The algorithm progresses with two primary classes of objectives. Graph based objectives preserve the topological properties of complexes whereas $\mathrm{GO}$ based semantic similarity between protein pairs control the accumulation of functionally similar proteins in the same cluster. Moreover the predicted complexes show consistently better result in context of some performance metrics.

We have also built an association between predicted protein complexes and 22 primary key disease classes to study the relationship between complexes and disorders associated with these classes. For finding the association of predicted complexes in different disease classes, we have extensively searched the involvement of proteins implicated by different disorders in the predicted complexes and built two bipartite networks between complexes and disorders. Interestingly, we have found most of the predicted complexes are associated with disorders belonging to the 'Cancer' disease class. Additionally, the disorders belonging to 'Endocrine' and 'multiple' disease classes have also shared a significant proportion of proteins involved in multiple predicted complexes.

Protein complexes are now considered as potential targets for intervention of new therapeutics to treat against new diseases. It is possible to integrate the drug-disease association information along with the complex-disease association. The new paradigm in drug discovery analysis is now given emphasis on the polypharmacological properties of drugs. Polypharmacological drugs are generally targets multiple cellular function for the treatment of complex diseases. By incorporating protein complexes with drug-disease association, it may be possible to uncover some relationship between protein complexes and targeted drugs.

Moreover we can potentially merge the time series gene expression profiles affected by a specific disease with our complex-disease bipartite network structure. This can offer a new way to exploit new topological features and complex modular structure in protein complex-disease and protein complex-disease-drug network. We are now working in this direction.

\section{Additional files}

Additional file 1. The human protein-protein interaction data.

Additional file 2. The source code of the proposed methodology.

Additional file 3. The processed data for constructing complex-disease bipartite network and ROC plot.

Additional file 4: Table S1. The predicted protein complexes and their association with multiple disorders corresponding to the specific disease classes.

\section{Authors' contributions}

SR did the initial planning and collected the dataset. AM and SR performed the data processing, developed the code, performed the analysis, and drafted the manuscript. SB and UM provided constructive discussion, corrected the manuscript, and supervised the complete work. All authors read and approved the final manuscript.

\section{Author details}

${ }^{1}$ Machine Intelligence Unit, Indian Statistical Institute, Kolkata 700108, West Bengal, India. ${ }^{2}$ Department of Computer Science and Engineering, Aliah University, Kolkata 700156, West Bengal, India. ${ }^{3}$ Department of Computer Science and Engineering, University of Kalyani, Kalyani 741235, West Bengal, India. ${ }^{4}$ Department of Computer Science and Engineering, Jadavpur University, Kolkata 700032, West Bengal, India.

\section{Acknowledgements}

AM gratefully acknowledges the support from DST PURSE scheme.

Compliance with ethical guidelines

Competing interests

The authors declare that they have no competing interests. 
Received: 3 December 2014 Accepted: 28 July 2015

Published online: 09 August 2015

\section{References}

1. Mirny L, Spirin V (2003) Protein complexes and functional modules in molecular networks. Proc Natl Acad Sci 100(21):12123-12128

2. Altaf-Ul-Amin M, Shinbo Y, Mihara K, Kurokawa K, Kanaya S (2006) Development and implementation of an algorithm for detection of protein complexes in large interaction networks. BMC Bioinform 7:207

3. Brohee S, van Helden J (2006) Evaluation of clustering algorithms for protein-protein interaction networks. BMC Bioinform 7:471-488

4. Pereira-Leal JB, Enright AJ, Ouzounis CA (2004) Detection of functional modules from protein interaction networks. Proteins 54:49-57

5. Bader G, Hogue C (2003) An automated method for finding molecular complexes in large protein interaction networks. BMC Bioinform 4:1471-2105

6. Nepusz T, Yu H, Paccanaro A (2012) Detecting overlapping protein complexes from protein-protein interaction networks. Nat Methods 9:471-472

7. Frey BJ, Dueck D (2007) Clustering by passing messages between data points. Science 315:972-976

8. Mukhopadhyay A, Ray S, De M (2012) Detecting protein complexes in a PPI network: a gene ontology based multi-objective evolutionary approach. Mol Biosyst 8(11):3036-3048

9. Zhao J, Lee S, Huss M, Holme P (2012) The network organization of cancer-associated protein complexes in human tissues. Nature Scientific Reports. doi:10.1038/srep01583

10. Lea D, Uyb N, Dungc P, Binhc H, Kwond Y (2013) Towards the identification of disease associated protein complexes. Proced Comput Sci 23:15-23

11. Vanunu O, Magger O, Ruppin E, Shlomi T, Sharan R (2010) Associating genes and protein complexes with disease via network propagation. PLoS Comput Biol. doi: 10.1371/journal.pcbi.1000641

12. Huang D, Sherman B, Tan Q, Collins J, Alvord W, Roayaei J et al (2007) The david gene functional classification tool: a novel biological modulecentric algorithm to functionally analyze large gene lists. Genome Biol 8(9):183

13. Schlicker A, Domingues F, Rahnenfuhrer J, Lengauer T (2006) A new measure for functional similarity of gene products based on gene ontology. BMC Bioinform 7:302

14. Deb K, Pratap A, Agrawal S, Meyarivan T (2002) A fast and elitist multiobjective genetic algorithm: NSGA-II. IEEE Trans Evol Comput 6:182-197

15. Deb K (2001) Multi-objective optimization using evolutionary algorithms. John Wiley and Sons Ltd, England

16. Bandyopadhyay S, Mukhopadhyay A, Maullik U (2011) Multiobjective genetic algorithms for clustering-applications in data mining and bioinformatics. Springer, Berlin, Heidelberg. ISBN 978-3-642-16614-3

17. Peri S, Navarro J, Kristiansen T, Amanchy R, Surendranath V, Muthusamy B et al (2004) Human protein reference database as a discovery resource for proteomics. Nucleic Acids Res 32:497-501

18. Leung H, Xiang Q, Yiu S, Chin F (2009) Predicting protein complexes from PPI data: a core-attachment approach. J Comput Biol 16(2):133-144

19. Wu M, Xiaoli L, Chee-Keong K, See-Kiong N (2009) A core-attachment based method to detect protein complexes in ppi networks. BMC Bioinform 10(169):10-11861471210510169

20. King AD, Przulj N, Jurisica I (2004) Protein complex prediction via costbased clustering. Bioinformatics 20:3013-3020

21. Srihari S, Ning K, Leong HW (2010) MCL-CAw: a refinement of mcl for detecting yeast complexes from weighted ppi networks by incorporating core-attachment structure. BMC Bioinform 11(504):10-11861471210511504
22. Zaki N, Efimov D, Berengueres J (2013) Protein complex detection using interaction reliability assessment and weighted clustering coefficient. BMC Bioinform 4(163):10-11861471210514163

23. Goh K, Cusick M, Valle D, Childs B, Vidal M, Barabasi A (2007) The human disease network. PNAS 104:8685-8690

24. Prelic A (2006) A systematic comparison and evaluation of biclustering methods for gene expression data. Bioinformatics 22:1122-1129

25. Wang H, Azuaje F, Bodenreider O, Dopazo J (2004) Gene expression correlation and gene ontology-based similarity: an assessment of quantitative relationships. In: Proceedings of IEEE symposium on computational intelligence in bioinformatics and computational biology, pp 25-31

26. Kikugawa S, Nishikata K, Murakami K, Sato Y, Suzuki M, Altaf-Ul-Amin M et al (2012) Pcdq: human protein complex database with quality index which summarizes different levels of evidences of protein complexes predicted from h-invitational protein-protein interactions integrative dataset. BMC Syst Biol 6:7

27. Brohee S, van Haden J (2006) Evaluation of clustering algorithms for protein--protein interaction networks. BMC Bioinform 7:488

28. Takada I, Kouzmenko A, Kato S (2009) Wnt and PPAR gamma signaling in osteoblastogenesis and adipogenesis. Nat Rev Rheumatol 5(8):442-7

29. Najdi R, Holcombe R, Waterman M (2011) Wht signaling and colon carcinogenesis. J Carcinog 10:5. doi:10.4103/1477-3163.78111

30. Bienz M, Clevers H (2000) Linking colorectal cancer to wnt signaling. Cell 103:311-320

31. Giles RH, van Es JH, Clevers H (2003) Caught up in a wnt storm: wnt signaling in cancer. Biochim Biophys Acta 1653(1):1-24

32. Moustakas A, Souchelnytskyi S, Heldin C (2001) Smad regulation in tgf- $\beta$ signal transduction. J Cell Sci 114:4359-4369

33. Kajiguchi T, Simokawa T, Saito M, Takeyama H (2000) Transformation of polycythemia vera to acute promyelocytic leukemia. Int J Hematol 4(72):520-521

34. Kuno Y, Takeo T, Kawashima K (1997) Cd7+, cd34+, electronmicroscopically peroxidase-negative acute leukemia transformed from polycythemia vera after 12 years follow-up. Jpn J Clin Hematol 97(38):782-787

35. Bayle P, Bazex J, Lamant L, Lauque D, Durieu C, Albes B (2004) Multiple perforating and non perforating pilomatricomas in a patient with ChurgStrauss syndrome and Rubinstein-Taybi syndrome. J Eur Acad Dermatol Venereol 5(18):607-610

36. Seda C, Salas A, Sanchez C, Blasco J, Garcia I, Sanchez J et al (2011) Thrombocytosis and hematocrit as prognostic factors in renal carcinoma. Arch Esp Urol 64(9):883-890

37. Kawamura Y, Kazama S, Miyahara T, Masaki T, Muto T (1999) Sigmoid colon cancer associated with primary hyperparathyroidism: report of a case. Surg Today 29(8):789-790

38. Micale S, Kane M, Busch R (2012) Spontaneous resolution of primary hyperparathyroidism in parathyroid adenoma. Case Rep Endocrinol 10:793753

39. Wei B, Shen $H$, Wang J, Xing $X$, Tong G, Chang H et al (2012) Surgical treatment of hyperparathyroidism due to parathyroid tumors. Chin $J$ Otorhinolaringol 47(10):861-4

\section{Submit your next manuscript to BioMed Central and take full advantage of:}

- Convenient online submission

- Thorough peer review

- No space constraints or color figure charges

- Immediate publication on acceptance

- Inclusion in PubMed, CAS, Scopus and Google Scholar

- Research which is freely available for redistribution

Submit your manuscript at

www.biomedcentral.com/submit 